International Journal of Pure and Applied Mathematics

Volume 108 No. 1 2016, 13-21

ISSN: 1311-8080 (printed version); ISSN: 1314-3395 (on-line version)

url: http://www.ijpam.eu

doi: 10.12732 /ijpam.v108i1.3

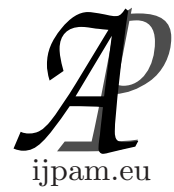

\title{
NON-EXISTENCE OF BIANCHI TYPE IV SPACE-TIME IN MODIFIED GRAVITY
}

\author{
B. Mishra ${ }^{1 \S}$, P.K. Sahoo ${ }^{2}$, Avinash Aman ${ }^{3}$ \\ 1,2,3 Department of Mathematics \\ Birla Institute of Technology and Science-Pilani \\ Hyderabad Campus, Hyderabad, 500078, INDIA
}

\begin{abstract}
We have investigated non-diagonal Bianchi type IV space-time filled with perfect fluid in the framework of $f(R, T)$ gravity, where $R$ is the Ricci scalar and $T$ is the trace of the energy-momentum tensor. We have considered two cases of $f(R, T)$ gravity: $f(R, T)=$ $R+2 f(T)$ and $f(R, T)=f_{1}(R)+f_{2}(T)$. In both the cases, it is found that the perfect fluid does not survive and the space-time turns out to be flat in $f(R, T)$ theory of gravity.
\end{abstract}

Key Words: space-time, $f(R, T)$ gravity, perfect fluid

\section{Introduction}

Einstein's general theory of relativity generalizes relativity and gravity, giving combined knowledge of gravity as a geometric property of the space-time and is the basis for most of the known gravitational phenomena. But the recent cosmological data brings a new understanding on cosmology. It indicates to us that the universe is expanding with constant acceleration (Riess et al. 1998; Perlmutter et al. 1999 and Bennet et al. 2003) and 'Dark Energy' is believed to be its source whose origin is still a mystery in modern cosmology. Dark energy dominates the universe and is the component with negative pressure constituting $68.3 \%$ of the critical density. The most appropriate candidate for explaining the cosmic acceleration is "The Cosmological Constant", but it faces many problems due to large discrepancies between observations and theory(Copeland et al. 2006; Nojiri and Odintsov 2007).

Received: February 24, 2016

Published: May 27, 2016

§ Correspondence author (c) 2016 Academic Publications, Ltd. url: www.acadpubl.eu 
New data-sets such as Cosmic Microwave Background Radiation (CMBR) and supernovae surveys indicate that the energy composition of the universe is as follows: $68.3 \%$ Dark energy, and $4.9 \%$ ordinary baryonic matter and the remaining is the dark matter(Riess et al. 2004; Eisenstein et al. 2005; Astier et al. 2006; Spergel et al. 2007). Hence, recently dark energy models are created by modifying the geometrical part of the Einstien-Hilbert action of general relativity.Some of the theoretical models proposed to explain the dark energy and accelerated expansion of the universe are Chaplygin gas (Kamenshchik et al. 2001; Bento et al. 2002), f-essence, k-essence, phantom energy (Caldwell 2002; Nojiri and Odintsov 2003), quintessence (Sahni and Starobinsky 2000; Sahni 2004; Padmanabhan 2008). Among all these models of dark energy, $f(R)$ theory of gravity(Caroll et al. 2004) and $f(G)$ Gauss-Bonnet gravity are the modified gravity theories. In recent years these theories are gaining more attraction. $f(R, T)$ gravity and $f(T)$ gravity(where $T$ is trace of the energy momentum tensor) are other approaches to modified gravity theory.Some of the authors who have worked on $f(R)$ gravity models are Chiba et al. (2007), Nojiri and Odintsov (2007), Multamaki and Vilja(2006,2007) and Shamir(2010). This modified gravity approach has been verified in successfully explaining the unification of early time inflation and late-time accelerated expansion of the universe.

Isotropic, homogeneous and expanding nature of the physical universe is confirmed by the analysis of the microwave background fluctuations and is well represented within the framework of the isotropic Friedman-RobertsonWalker(FRW) cosmology. In contrary other analysis reveal inconsistency. The WMAP data analysis supports the fact that the universe has a preferred direction and it should reach a slightly anisotropic geometry, making the study of anisotropic Bianchi models important. Thus making the investigations of Bianchi type models in $f(R, T)$ gravity an area of importance. Nojiri and Odintsov (2006) developed a general scheme for $f(R)$ reconstruction from realistic FRW cosmology. Adhav (2012) obtained Biachi type-I cosmological model in $f(R, T)$ gravity.Reddy et al (2012a) have discussed Bianchi Type-III cosmological model in $f(R, T)$ gravity whereas Reddy et al. (2013a), Reddy and Shanthikumar (2013a) studied Bianchi type-III dark energy model and some anisotropic cosmological models in $f(R, T)$ gravity,respectively. Mishra and Sahoo (2014) also discussed Bianchi type $V I_{h}$ perfect fluid cosmological model in $f(R, T)$ theory. Sahoo et al.(2014) have studied the dynamics of five dimensional Kaluza-klein metric for a perfect fluid distribution in the frame work of modified gravity. Out of this, Bianchi type-IV space-time in $f(R, T)$ gravity has not been considered in the literature.

Motivated by the above discussion and investigations in modified theories 
of gravity, in this paper, we propose to study Bianchi type-IV space time in $f(R, T)$ gravity by considering $f(R, T)=R+2 f(T)$ and $f(R, T)=f_{1}(R)+$ $f_{2}(T)$, where $f_{1}(R)=\lambda R$ and $f_{2}(T)=\lambda T$. This paper has been organized as follows: In Section 2 gravitational field equations in $f(R, T)$ gravity are derived,. Section 3 formulates the field equations for Bianchi type-IV spacetime in $f(R, T)$ gravity for both the cases and showing its non existence. Finally conclusions are summarized in the last Section 4.

\section{2. $\mathrm{f}(\mathrm{R}, \mathrm{T})$ Gravity}

The $f(R, T)$ modified theory of gravity is another extension of standard General Relativity, where the gravitational Lagrangian is given by an arbitrary function of Ricci scalar $R$ and of the trace of stress-energy tensor. In $f(R, T)$ gravity, Hilbert-Einstien type variational principle yields the gravitational field equations. The $f(R, T)$ modified gravity action as considered by Harko et al. (2011)is given by

$$
S=\frac{1}{16 \pi} \int f(R, T) \sqrt{-g} d^{4} x+\int L_{m} \sqrt{-g} d^{4} x,
$$

where $f(R, T)$ is an arbitrary function of Ricci scalar $R, T$ being the trace of the stress-energy tensor $\left(T_{i j}\right)$ of the matter and $L_{m}$ is the matter Lagrangian density. The stress-energy tensor of matter is defined as

$$
T_{i j}=\frac{-2}{\sqrt{-g}} \frac{\delta\left(\sqrt{-g} L_{m}\right)}{\delta g^{i j}}
$$

We assumed here that the dependence of matter Lagrangian is on the metric tensor $g_{i j}$ rather than its derivatives.

The trace of the energy tensor of matter is given by

$$
T=g^{i j} T_{i j}
$$

So in this case the stress-energy tensor of matter is

$$
T_{i j}=g_{i j} L_{m}-2 \frac{\partial L_{m}}{\partial g^{i j}}
$$

Varying the action $\mathrm{S}$ of the gravitation field with respect to the metric tensor components $g^{i j}$, the field equations of $f(R, T)$ gravity are obtained as follows

$$
f(R, T) R_{i j}-\frac{1}{2} f(R, T) g_{i j}+\left(g_{i j} \square-\nabla_{i} \nabla_{j}\right) f_{R}(R, T)
$$




$$
=8 \pi T_{i j}-f_{T}(R, T) T_{i j}-f_{T}(R, T) \theta_{i j}
$$

where

$$
\theta_{i j}=-2 T_{i j}+g_{i j} L_{m}-2 g^{\alpha \beta} \frac{\partial^{2} L_{m}}{\partial g^{i j} \partial g^{\alpha \beta}}
$$

Now here $f_{R}(R, T)=\frac{\partial f(R, T)}{\partial R}, f_{T}(R, T)=\frac{\partial f(R, T)}{\partial T}, \square \equiv \nabla^{i} \nabla_{i}$ where $\nabla_{i}$ is the covariant derivative and $T_{i j}$ is the standard matter energy-momentum tensor. Contraction of (5)yields

$$
f_{R}(R, T) R+3 \square f_{R}(R, T)-2 f(R, T)=8 \pi T-f_{T}(R, T)(T+\theta)
$$

where $\theta=\theta_{i}^{i}$. The above equation (7) gives a relation between the trace $T$ of energy-momentum tensor and Ricci scalar $R$.

It can be seen that when $f(R, T) \equiv f(R)$, eqn (5) yields the field equations of $f(R)$ gravity.

Now using (6), we get the variation of stress-energy. As there is no unique definition of matter Lagrangian, the matter Lagrangian can be taken as $L_{m}=p$. Now using the Lagrangian $L_{m}$, the stress-energy tensor of matter is given by

$$
T_{i j}=(\rho+p) u_{i} u_{j}+p g_{i j}
$$

where $u^{i}=(0,0,0,1)$ is the four velocity vector in the co-moving coordinate system such that $u^{i} u_{i}=1$ and $u^{i} \nabla_{j} u_{i}=0, \rho$ and $\mathrm{p}$ are energy density and pressure of the fluid respectively.

Then using eqn. (6), we obtain the variation of Stress-energy of perfect fluid as

$$
\theta_{i j}=-2 T_{i j}-p g_{i j}
$$

On the physical nature of the matter field,the field equations also depend through the tensor $\theta_{i j}$.

Hence in the case of $f(R, T)$ gravity depending on the nature of matter source. several theoretical models for different matter contributions for $f(R, T)$ gravity are available in literature. Harko et al (2011) gave three classes of model as

$$
f(R, T)=\left\{\begin{array}{l}
R+2 f(T) \\
f_{1}(R)+f_{2}(T) \\
f_{1}(R)+f_{2}(R) f_{3}(T)
\end{array}\right.
$$

Here we focus on the first two cases i.e. $f(R, T)=R+2 f(T)$ and $f(R, T)=$ $f_{1}(R)+f_{2}(T)$ where $f(T)$ is an arbitrary function of Stress-Energy tensor of matter. Now from (5) we get the field equations of $f(R, T)$ gravity as

$$
R_{i j}-\frac{1}{2} R g_{i j}=8 \pi T_{i j}-2 f^{\prime}(T) T_{i j}-2 f^{\prime}(T) \theta_{i j}+f(T) g_{i j}
$$


where prime denotes differentiation with respect to the argument.

In perfect fluid the field equations become

$$
R_{i j}-\frac{1}{2} R g_{i j}=8 \pi T_{i j}+2 f^{\prime}(T) T_{i j}+\left[2 p f^{\prime}(T)+f(T)\right] g_{i j}
$$

\section{Field Equations and its solutions}

We have considered a non diagonal Bianchi type $I V$ space-time of the form

$$
d s^{2}=-d t^{2}+A^{2} d x^{2}+B^{2} e^{2 x} d y^{2}+c^{2} e^{2 x}[x d y+d z]^{2}
$$

where $A(t), B(t)$ and $C(t)$ are three anisotropic directions of the space and functions of cosmic time only.

These functions are not equal due to radial asymmetry. The matter tensor can be defined as

$$
\theta_{i j}=-2 T_{i j}-p g_{i j}=(\rho,-p,-p,-p)
$$

\subsection{Case I}

As a first case we consider the functional $f(R, T)$ in the form $f(R, T)=R+$ $2 f(T)$. Again, we chose $f(T)=\lambda T$ where $\lambda$ is a constant. The field equations for Bianchi type $I V$ space-time in $f(R, T)$ gravity are obtained as

$$
\begin{gathered}
\frac{\ddot{B}}{B}+\frac{\ddot{C}}{C}+\frac{\dot{B} \dot{C}}{B C}-\frac{1}{A^{2}}+\frac{C^{2}}{4 A^{2} B^{2}}=(8 \pi+3 \lambda) p-\lambda \rho \\
\frac{2 \dot{A}}{A}-\frac{\dot{B}}{B}-\frac{\dot{C}}{C}=0 \\
C^{2} x^{2}\left[\frac{2}{A^{2} x}+\frac{1}{4 A^{2} x^{2}}+\frac{\ddot{A}}{A}+\frac{\ddot{B}}{B}+\frac{\dot{A} \dot{B}}{A B}-\frac{1}{A^{2}}-\frac{3 C^{2}}{4 A^{2} B^{2}}\right]+B^{2}\left[\frac{\ddot{A}}{A}+\frac{\ddot{C}}{C}+\frac{\dot{A} \dot{C}}{A C}-\frac{1}{A^{2}}\right] \\
=\left[B^{2}+C^{2} x^{2}\right][(8 \pi+3 \lambda) p-\rho] \\
\frac{\ddot{A}}{A}+\frac{\ddot{B}}{B}+\frac{\dot{A} \dot{B}}{A B}-\frac{1}{A^{2}}-\frac{3 C^{2}}{4 A^{2} B^{2}}=(8 \pi+3 \lambda) p-\lambda \rho \\
\frac{1}{A^{2} x}-\frac{1}{A^{2}}-\frac{3 C^{2}}{4 A^{2} B^{2}}+\frac{\ddot{A}}{A}+\frac{\ddot{B}}{B}+\frac{\dot{A} \dot{B}}{A B}=(8 \pi+3 \lambda) p-\lambda \rho \\
\frac{\dot{A} \dot{B}}{A B}+\frac{\dot{B} \dot{C}}{B C}+\frac{\dot{A} \dot{C}}{A C}-\frac{3}{A^{2}}-\frac{C^{2}}{4 A^{2} B^{2}}=-(8 \pi+3 \lambda) \rho+\lambda p
\end{gathered}
$$


where an overhead dot represents differentiation with respect to cosmic time ' $t$ '.

On integration equation (17) yields

$$
A^{2}=k(B C)
$$

without loss of generality we take $k=1$,subsequently equation (22) reduces to

$$
A^{2}=B C
$$

Now from equations (19) and (20), we get

$$
\frac{1}{A^{2} x}=0
$$

which leads to inconsistency for both the cases $A$ is very large or $x=\infty$. Neither of the cases is acceptable under geometrical and physical ground. Thus the $f(R, T)$ gravity is not compatible in Bianchi type $I V$ space-time.

\subsection{Case II}

In this case, we consider $f(R, T)$ in the form

$$
f(R, T)=f(R)+f(T)
$$

where $f(R)$ and $f(T)$ are some functions of the Ricci scalar $R$ and the stress energy tensor $T$. In principle one can chose any functional form for $f(R)$ and $f(T)$ to get viable cosmological solutions. Our interest in the present work is to investigate whether or not the present gravity model can be sustained for Bianchi type $I V(\mathrm{BIV})$ metric. We have already shown that in the first case, the present modified gravity model is incompatible for BIV metric. In view of this we chose simple forms of $f(R)$ and $f(T)$ such as $f(R)=\alpha R$ and $f(T)=\alpha T$. Here $\alpha$ is a constant.

Now the corresponding field equations for the non-diagonal Bianchi type IV space-time are obtained as:

$$
\begin{gathered}
\frac{\ddot{B}}{B}+\frac{\ddot{C}}{C}+\frac{\dot{B} \dot{C}}{B C}-\frac{1}{A^{2}}+\frac{C^{2}}{4 A^{2} B^{2}}=\left(\frac{8 \pi+\alpha}{\alpha}\right) p+\Lambda \\
\quad \frac{2 \dot{A}}{A}-\frac{\dot{B}}{B}-\frac{\dot{C}}{C}=0 \\
C^{2} x^{2}\left[\frac{2}{A^{2} x}+\frac{1}{4 A^{2} x^{2}}+\frac{\ddot{A}}{A}+\frac{\ddot{B}}{B}+\frac{\dot{A} \dot{B}}{A B}-\frac{1}{A^{2}}-\frac{3 C^{2}}{4 A^{2} B^{2}}\right]+B^{2}\left[\frac{\ddot{A}}{A}+\frac{\ddot{C}}{C}+\frac{\dot{A} \dot{C}}{A C}-\frac{1}{A^{2}}\right]
\end{gathered}
$$




$$
\begin{gathered}
=\left[B^{2}+C^{2} x^{2}\right]\left[\left(\frac{8 \pi+\alpha}{\alpha}\right) p+\Lambda\right] \\
\frac{\ddot{A}}{A}+\frac{\ddot{B}}{B}+\frac{\dot{A} \dot{B}}{A B}-\frac{1}{A^{2}}-\frac{3 C^{2}}{4 A^{2} B^{2}}=\left(\frac{8 \pi+\alpha}{\alpha}\right) p+\Lambda \\
\frac{1}{A^{2} x}-\frac{1}{A^{2}}-\frac{3 C^{2}}{4 A^{2} B^{2}}+\frac{\ddot{A}}{A}+\frac{\ddot{B}}{B}+\frac{\dot{A} \dot{B}}{A B}=\left(\frac{8 \pi+\alpha}{\alpha}\right) p+\Lambda \\
\frac{\dot{A} \dot{B}}{A B}+\frac{\dot{B} \dot{C}}{B C}+\frac{\dot{A} \dot{C}}{A C}-\frac{3}{A^{2}}-\frac{C^{2}}{4 A^{2} B^{2}}=\left(\frac{8 \pi+\alpha}{\alpha}\right) \rho-\Lambda
\end{gathered}
$$

where $\Lambda=\frac{\rho-p}{2}$.

One can note from equations (29) and (30), a similar situation as that of case-I is obtained. The field equations lead to quite unphysical situation implying an infinitely large value for either the metric potential $A$ or $x$. This leads to a cosmological inconsistency.

It is not that, the two cases that we have considered in the work, with the specific forms of $f(R)$ and $f(T)$ in the given modified gravity model as proposed by Harko et al. and a specific matter field lead to inconsistent cosmological solutions with BIV metric. This is true for whatever matter field we will consider to get some cosmological implications. This is quite clear from equation (12), where one can see that for some coordinates, the right hand side becoming the same and in the left hand side there will be an extra term like $\frac{1}{A^{2} x}$ in one equation. This appearance of an extra scale dependent term leads to the inconsistency. It is worth to mention here that, Harvey et al.(1977) have investigated BIV metric with a different spatial geometry in general relativity for perfect fluid distribution and found that only vacuum solution can be possible for this metric. they also extended their work with the inclusion of electromagnetic contribution to get similar results. In an earlier work (Mishra2015), we have investigated the compatibility of the BIV metric in general relativity to show that the model is quite incompatible. The present result obviously warns of proper care while cosmological implications are sought with BIV metric.

\section{Conclusions}

We have intended to develop the $f(R, T)$ gravity in a space-time governed by a non-diagonal Bianchi type $I V$ metric. However, this gravity is not compatible in Bianchi type $I V$ space-time. This may be due to the fact that the geometry of the space-time is governed by non-diagonal metric. Harvey and Tsoubelis 
(1977)have considered a different form of Bianchi type $I V$ metric and they suggested that only vacuum solution can be obtained from it. However, in our case, we can not even obtain the vacuum solution also. The field equations lead to completely unphysical states because of the term $1 /\left(A^{2} x\right)$. The outcome is similar as one of our previous study on dark energy[Mishra et al., 2015].Therefore this study may be pursued further to understand the development of $f(R, T)$ gravity in non-diagonal space-time in general. Moreover, the reason need to be found out why $f(R, T)$ gravity is showing this kind of behaviour in this space time i.e whether it is the problem of the space time or the formulation of $f(R, T)$ gravity.

\section{Acknowledgement}

BM acknowledge SERB-DST, New Delhi, India for financial support to carry out the Research project (No. SR/S4/MS:815/13).

\section{References}

[1] K.S. Adhav, Astrophys. Space Sci., 339, No. 365 (2012).

[2] P. Astier, et al, Astron. Astrophys., 447, No. 31 (2006).

[3] C.L. Bennet, et al, Astrophys. J. Suppl. Ser., 148, No. 1 (2003).

[4] M.C. Bento, O. Bertolami, A.A. Sen, Phys. Rev. D, 66, 043507 (2002).

[5] R.R. Caldwell, Phys. Lett B, 545, No. 23 (2002).

[6] S.M. Caroll, V. Duvuri, et al, Phys. Rev. D, 70, 043528 (2004).

[7] T. Chiba, L. Smith, A.L. Erickcek, Phys. Rev. D, 75, 124014 (2007).

[8] E.J. Copeland, et al, Int. J. Mod. Phys. D, 15, No. 1753 (2006).

[9] D.J. Eisenstein, et al, Astron. J., 633, No. 560 (2005).

[10] A. Harvey, D. Tsoubelis, Phys. Rev. D, 15 (1977), 2734-2737.

[11] T. Harko, F.S. Lobo, S. Nojiri, S.D. Odintsov, Phys. Rev. D, 84, 024020 (2011).

[12] A.Y. Kamenshchik, M. Moschella, V. Pasquier, Phys. Lett. B, 511, No. 265 (2001).

[13] B. Mishra, P.K. Sahoo, Astrophys and Space Sci., 352 (2014), 331-336.

[14] B. Mishra, P.K. Sahoo, Srikanth Suresh, Astrophys and Space Sci., 358, No. 7 (2015).

[15] T. Multamaki, I. Vilja, Phys. Rev. D, 74 (2006), 064022.

[16] T. Multamaki, I. Vilja, Phys. Rev. D, 76 (2007), 064021.

[17] S. Nojiri, S.D. Odintsov, Phys. Rev. D, 68 (2003), 123512.

[18] S. Nojiri, S.D. Odintsov, ArXiv: hep-Th/0601213 (2006). 
[19] S. Nojiri, S.D. Odintsov, Int. J. Geom. Methods Mod. Phys., 4, No. 115 (2007); ArXiv: hep-Th/0601213.

[20] T. Padmanabhan, Gen. Rel. Grav., 40 (2008).

[21] S. Perlmutter, Astrophys. J., 517 (1999).

[22] D.R.K. Reddy, R. Santikumar, Astrophys. Space Sci., 344 (2013), 253.

[23] D.R.K. Reddy, R. Santikumar, Pradeep P.V. Kumar, Int. J. Theor. Phys., 52 (2013), 239.

[24] A.G. Riess, et al, Astron. J., 116 (1998), 1009.

[25] A.G. Riess, et al, (Supernova Search Team), Astron. J., 607 (2004), 665.

[26] V. Sahni, ArXiv: astro-ph/0403324v3 (2004).

[27] V. Sahni, A. Starobinsky, Int. J. Mod. Phys. D, 9 (2000), 373.

[28] M.F. Shamir, Astrophys. Space Sci., 330, 183.

[29] D.N. Spergel, et al, (WMAP), Astrophys. J. Suppl. Ser., 170 (2007), 3771. 
